\title{
TIME-OF-FLIGHT MEASUREMENTS OF ABSOLUTE BEAM ENERGY IN THE FERMILAB LINAC
}

\author{
M.B. Popovic, T.L. Owens, T.K. Kroc, I.J. Allen and C.W. Schmidt \\ Fermi National Accelerator Laboratory*
}

\begin{abstract}
Measurements have been made of the absolute energy of the fourth tank of our linac by measuring the time-of-flight of individual beam bunches passing between strip-line beam monitors at the entrance and exit of each tank of the linac. Accurate time-of-flight measurements are facilitated by the fact that the beam remains well bunched between strip-line monitors in the drift region beyond the fourth tank. Cable lengths were carefully measured using time-domain reflectometry. The data for tank 4 gave the time-of-flight over a 49.17 meter drift as $395.8( \pm 0.5)$ nanoseconds. This translates into a measured $\beta$ of 0.4155 . The design[1] $\beta$ for tank 4 is 0.4141 . Futher measurements on other linac tanks are planned, if machine time becomes available. In addition to determination of absolute beam energy, the time-of-flight data can be used for absolute energy calibration of the magnetic spectrometer system used for detailed energy analysis of the beam. We plan to implement this technique on the Linac Upgade[2] to be installed in the summer of 1993.
\end{abstract}

\section{EXPERIMENTAL CONFIGURATION}

In this experiment it was important to identify signals produced from the same bunch at two different locations. The macropulse (train of beam bunches) for the Fermilab linac is produced by an electrostatic chopper which selects a portion of the DC beam from the preaccelerator. The initial state has one plate charged and one grounded which deflects the DC beam into a carbon disk. When beam is desired, the charged plate is grounded and the beam passes undeflected. To terminate the macropulse, the second plate is taken to the opposite polarity of the first plate so the beam is again deflected. The risc time of the thyratrons that switch the plates is such that the shortest macropulse is 50 to $100 \mathrm{nsec}$ long which the buncher converts into approximatcly 10-20 micropulses.

A new circuit was devised out to produce the 3-4 micropulse burst used in these experiments. In the new cicuit, one of the plates is disconnected from its corresponding thyratron and grounded. The other plate is given a $\mathrm{DC}$ bias so that its starting voltage is half its original value. When the thyratron fires, the voltage on the plate switches polarity. As the voltage passes through zero, a short section of beam $(\sim 20$ nsec) passes down the beam line. The buncher then converts

\footnotetext{
* Fermilab is operated by the Universities Research Association under contract to the US Department of Energy.
}

the section of beam into 3 or 4 micropulses. Future experiments will attempt to produce single bunch micropulses by retuning the beamline and throught strategic placement of apertures.

Figure 1 is an oscillogram from a Tek-7104 oscilloscope screen. The pulse train contains seven bunches. The larger amplitude pulse traincomes from seven bunches pass

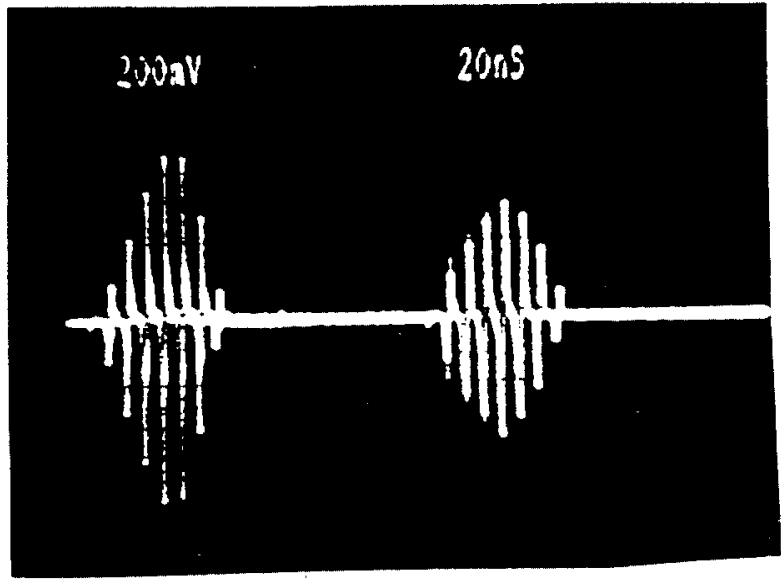

Figure 1.

ing the BPM detector at the entrance to tank 5 . The second pulse train is produced by the same seven bunches passing BPM detector located at exit of the tank 7 .

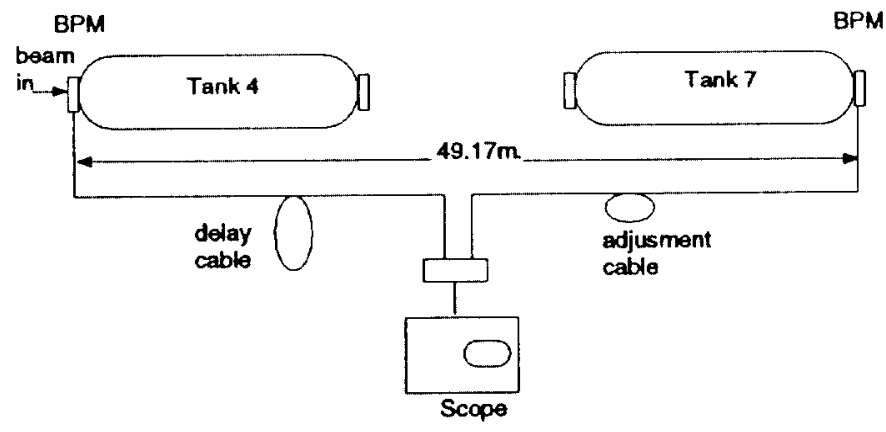

Figure 2.

The setup used in this experiment is shown schematically in figure 2 . The delay and adjustment cables are used to insure that signals from two BPM detectors arrive at about the same time and that they can both be displayed on the scope screen with the shortest available time-base. This experiment was performed using a Tek-7401 scope. The timebase was 2 nsec/div. The relative distance between two de- 
tectors is known with a accuracy of $\pm 5 \mathrm{~cm}$. We were able to read time interval between two signals with error of \pm 0.5

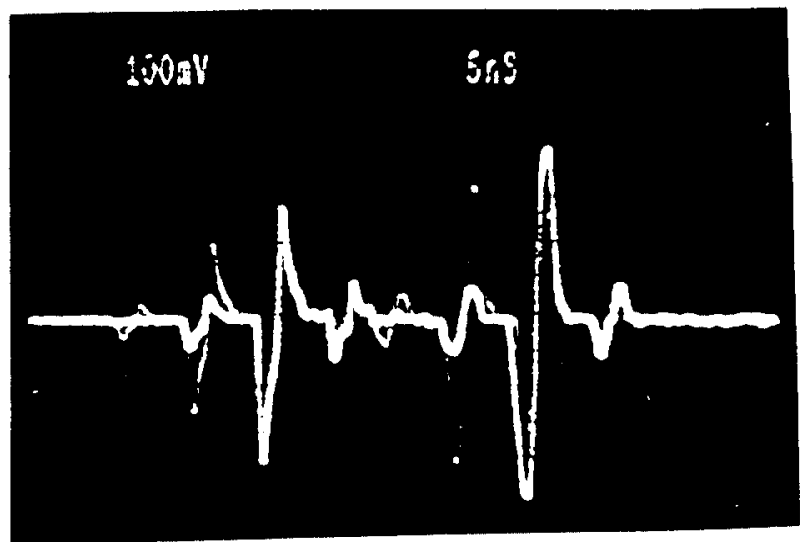

Figure 3.

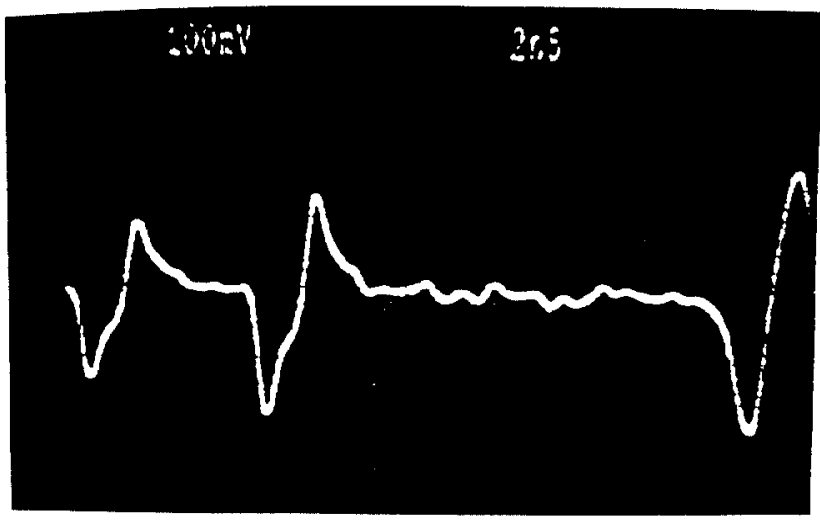

Figure 4.

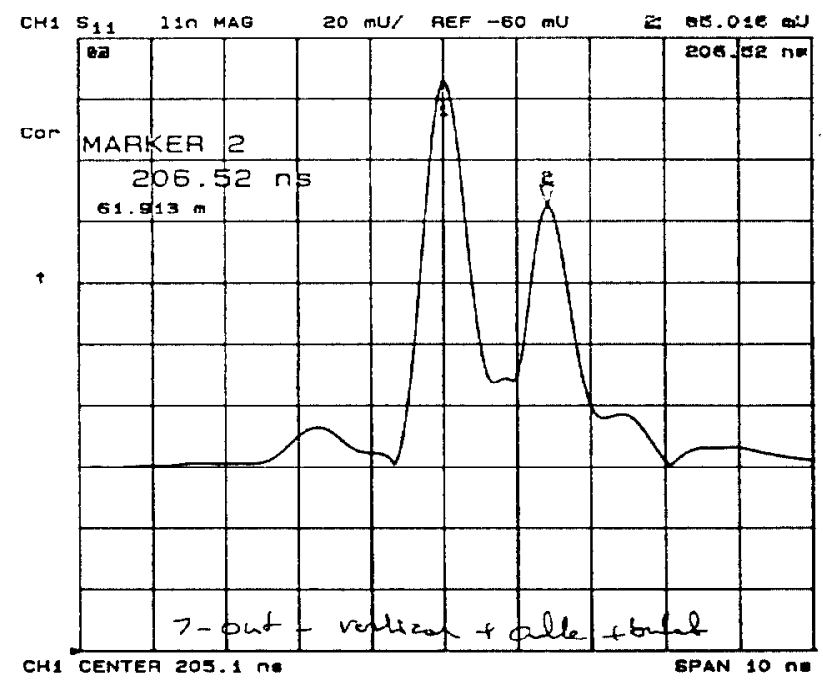

Figure 5.

nsec. Figures 3 and 4 show two signals on the scope screen with 5 nsec/div and $2 n s e c / d i v$ time bases, respectively. Data from figure 4 were used to determine $\beta$ at exit of the tank 4 .

The cable length or the time that the signal travels from BPM detector to the scope is measured using timedomain reflectometry. We have used an HP8753C network analyzer to determine cable length. Figure 5 . is typical example of cable length measurement. Three peaks are associated with the cable connector on the detector, gap of the strip-line detector and termination of the strip-line, respectively. The marker position represents the time it takes for an impulse launched at the test port to reach a discontinuity and to return. Although the position of the marker is displayed with tenths of picosecond, HP documentation[3] is not clear concerning how errors are estimated. Using short cables whose lengths are known we have convince our self that errors are less than \pm 0.5 nanosecond.

\section{RESULTS AND CONCLUSIONS}

The data for tank 4 gave the time-of-flight over a $49.17( \pm 0.05)$ meter drift as $395.8( \pm 0.5)$ nanoseconds. This translates into a measured $\beta$ of $0.4155[1 \pm 0.002]$. The design $\beta$ for tank 4 is 0.4141 . We plan to implement this technique on the Linac Pugged to be installed in the summer of 1993. We will use wall current monitors for bcam detection. Positions of detectors will be known to $\pm 0.5 \mathrm{am}$. We hope to measure time intervals up to \pm 0 .insect using a Tetroxide Schedule transient waveform recorder.

\section{REFERENCES}

[1] National Accelerator Laboratory, Design Report, July 1968.

[2] CA.We. Schmidt, "The Forelimb 400-MeV Linac Upgrade", this conference.

[3] HP8753C, "Operating Manual", Oct. 1989. 